\title{
A CONTEMPORARY RECAPITULATION OF MAJOR FINDINGS ON VEHICLE ROUTING PROBLEMS : MODELS AND METHODOLOGIES
}

\author{
D. G. N. D. Jayarathna, G. H. J. Lanel, Z.A.M.S. Juman
}

\begin{abstract}
In introdusing and designing innovative solutions to the problems related to transportation and distribution systems is a contemporary area in logistics. The ultimate objective of this paper is to initiate a thought provoking discusion on Vehicle Routing Problems (VRP) along with its modifications or changes which incorporates recent model developments and improvements. Both in operational research and computer science, VRP is a combinatorial optimization issue researched at length. Capacitated Vehicle Routing Problem (CVRP), Vehicle Routing Problem with Time Windows (VRPTW), Vehicle Routing Problem with MultiDepot (MDVRP) and other variants are integral components of VRP. In recent times, the areas of VRP categorization has been further discussed, the common constraints have been summarized and model algorithms have been developed. In toto the future model implications of VRP are analyzed and further, it is predicted that the Intelligent Vehicle Routing Problem and Intelligent Heuristic Algorithm would be an important arena of future researches.
\end{abstract}

Key words: Capacitated Vehicle Routing Problem, Exact Methods, Meta-Heuristics, Multi-Depot Vehicle Routing Problem

\section{Introduction to vehicle routing problems}

VRP can be explained as the issue of figuring out the lowest cost delivery directions or paths from a depot to a set of geographically dispersed clients, with a focus to crosswise limitations. Distribution of products and services is done by VRP in Supply chain and logistics management backdrop. This is vital for distribution management and therefore should be regularly solved by transporters. There are some modifications to the VRP and are expressed grounded on the nature of the goods transporting, the service value and features of clients and vehicles. Dantzig \& Ramser (1959) initially presented "Truck Dispatching Problem", which deals with modeling a fleet of homogeneous trucks to assist the demand for oil of number of gas stations from a central hub along with lowest travelling distance. Contributions towards rerarranging this issue to a linear advanced optimization

\section{G. N. D. Jayarathna (Corresponding Author)}

Colombo International Nautical and Engineering College, Sri Lanka email: nuwan@ cinec.edu

Dr. G. H. J. Lanel, University of Sri Jayewardenepura, Sri Lanka email: ghjlanel@sjp.ac.lk

Dr. Z.A.M.S. Juman, University of Peradeniya, Sri Lanka.

email: jumanabdeen@yahoo.com problem which generally comes across in the domain of Supply chain and operational management. This can be further explained as the way of servicing a group of clients, geographically scattered around the central warehouse, utilizing the fleet of trucks with different capacities becoming VRP which is amongst the most broadly used phenomena in the field of Advanced Liner Programming.

The enhancement of some forms of VRPs were found together with strategies for calculating the shortest route. Goetschalckx (2011) defines VRP as a problem of deciding the shortest route from a vehicle that begins from one depot to ' $n$ ' number multiple destinations to address various types of customer needs. Every vehicle which has a particular capacity, begins at a depot and returns to the main depot and further every client could only be toured once. Further, VRP offers a different range of heuristics and meta - heuristics approaches, these are introduced in Laporte (2009) and contribution of Gendreau (2002) and Cordeau (2005). The VRP is broadly taken in to account since of its familiar use and its significance in designing effective modes for the minimization of transportation cost in distribution systems. Therefore, this paper aims to design an approximation of procedures which are suitable for discovering high quality solutions in restricted time frames in the mean while addressing the real life problematic circumstances which are described by huge vehicle fleets and influenced in a positive manner to logistics and distribution strategies.

Comtemporary VRP software is being used by many public, private and multinational companies, and in large variety of industry sectors and in particular, Coca-Cola Enterprises and Anheuser-Busch Inbev are generally significant. (Drexl, 2012; Partyka \& Hall, 2014). The VRP has increased exponentially at a rate of $6 \%$ consistently which creates a ubiquity to monitor the expansions in the area and to make a presence of a strong indication of which substitutions and solution approaches are comparatively novel.

\subsection{Basics of Capacitated vehicle routing problem} and its variations

The CVRP could be identified as one of the crucial problems in the combinatoric optimization with various real world applications in transportation, distribution, logistics and fulfilling an extension of VRP. The objective of CVRP is to discover suitable paths or routes with minimum cost for a fleet of vehicles with 
an important capacity located in a solitary depot, with the target of serving a set of clients under the minimum limitations such as, all the routes start and finish at the same depot, each and every client is visited exactly one time and cumulative demand of each and every single route or path does not surpass the volume of the vehicles (Laporte, 2002).

In particular varieties of the CVRP must comply with route duration limitation that constrains the lengths of the feasible routes. Dantzig \& Ramser (1959) and Clarke \& Wright (1964) suggests the foremost heuristic for this issue as the initial mathematical formulae development and algorithm for the result of the CVRP while a number of solution techniques have been published since today. Also, general surveys conducted by Toth \& Vigo (2002) and Laporte (2002) can be found for the same problem. The CVRP falls in to the classification of NP - hard problems which could be directly answered with little scaled occasions of an issue. Therefore, many academic researchers have focused on implementing heuristic algorithms to take care of these issues (Gendreau \& Potvin (2010), Laporte \& Ropke (2014). For a very long period, various investigations have been focused on taking care of these problems and several methodologies have been reached from direct tree hunt with Branch-andBound to column generation (Eilon \& Christofides, 1969).

\subsubsection{Mathematical formulation of CVRP}

Suppose $\mathrm{G}=\left(\mathrm{V}_{\mathrm{o}}, \mathrm{H}_{\mathrm{o}}\right)$ be a comprehensive absorbed graph,

where, $V_{o}=\{0,1,2, \ldots, m\}$ as the set of vertices

$\mathrm{H}_{\mathrm{o}}=\left\{(\mathrm{j}, \mathrm{k}): \mathrm{j}, \mathrm{k} \in \mathrm{V}_{\mathrm{o}}, \mathrm{j} \neq \mathrm{k}\right\}$ be as the set of

edges,

which first node $\left(0^{\text {th }}\right.$ node $)$ denotes the depot for a fleet of $\mathrm{p}$ trucks with the similar volume $\mathrm{Q}_{\mathrm{o}}$ and $\mathrm{m}$ vertices signify geographically spreaded clients. Every client $\mathrm{j} \in$ $\mathrm{V}_{\mathrm{o}}-\{0\}$ has a certain positive value demand $\mathrm{d}_{\mathrm{j}} \leq \mathrm{Q}_{\mathrm{o}}$. The non negative travel cost $c_{j k}$ is accompanying with each edge $(\mathrm{j}, \mathrm{k}) \in \mathrm{H}_{\mathrm{o}}$. The cost matrix for the given graph is symmetric, i.e. $c_{j k}=c_{k j}$ for all $\mathrm{j}, \mathrm{k} \in \mathrm{V}, \mathrm{j} \neq \mathrm{k}$ and fulfills the triangular inequality law , $\mathrm{c}_{\mathrm{jk}}+\mathrm{c}_{\mathrm{kp}} \geq \mathrm{c}_{\mathrm{jp}}$ for all $\mathrm{j}, \mathrm{k}, \mathrm{p} \in \mathrm{V}_{\mathrm{o}}$, (Toth \& Vigo ,2002).The least number of trucks required to assist set of all clients is $\left[\sum_{j=1}^{m} d_{j}\right] /$ Qo ]

\subsubsection{Formulation of Two - index vehicle flow}

In this formulation, $x_{j k}$ is a binary decision parameter assuming the value as 1 , if there is a traveling path that drives from client $j$ to $k$ straight, for all $j, k \in \mathrm{V}_{\mathrm{o}}$. In addition to that, $y_{k}$ is a continuous decision measure that is match to the sum of all demands on the journey that visits node $k \in \mathrm{V}_{\mathrm{o}}$ up to this traveling tour. With all these corresponding measurements and decision criteria, The two - index flow formalulation of the CVRP Munari et.al (2017) can given by :

$$
\begin{gathered}
\operatorname{Min} \sum_{j=0}^{m+1} \sum_{k=0}^{m+1}\left(c_{j k} x_{j k}\right)-------- \\
-------[1] \\
\sum_{k=1, k \neq j}^{m+1}\left(x_{j k}\right)=1, \text { for } j
\end{gathered}
$$

Limitation (2) ensures that all clients are visited precisely once. Limitation (3) ensures that the precise movement of trucks via the edges, by uttering that if a truck reaches to a node $h \in \mathrm{V}_{\mathrm{o}}$, then it should leave from this node. Extreme number of paths to $K_{o}$ and the number of trucks are the restrictions which given by limitation (4). Limitation (5) and (6) together affirms that the truck volume is not surpassed. The main purpose of this function is depicted by (1) and forces that the absolute travel cost value of the paths are minimalized. Limitation (5) also elude sub tours in the answer, for example cycling travel routes that do not cross through the central depot. Various kinds of limitations are suggested in major findings to execute truck volumes and/or elude sub tours Irnich et al. (2014). Polynomial number of restrictions are there in the model based on the number of clients and can be considered as the advantage of using constraint (5) and (6). According to Semet et al.(2014) the lower bound offered by the linear relaxation will be continued to capacity limitations, even though the collection of limitations becomes exponential in terms of the number of clients, necessitating the usage of outlet and cut approach.

\subsection{Introduction to Capacitated Vehicle Routing Problem with Time Windows (CVRPTW)}

The CVRPTW is the CVRP with some extra time window imperatives. The aim of the VRPTW is to help several clients inside predefined time windows at least cost (interms of distance travelled), referring the capacity and total tour time limitations for every truck. In real world, set of clients could be assisted restricted to a defined time frame which could state as the time windows only. Thus, time window limitations must be concentrated well . Clients start to be assisted inside a time window $\left[E_{j}, L_{j}\right]$. In the event that trucks arrive at client $j$ before $E_{j}$, vehicles could hold up until $E_{j}$, as no extra panelty cost to wait exists. As the client is not accessible, anything cannot be picked up by the truck and extra fine costs should be well concentrated in the event that trucks arrive at client $j$ after $L_{j}$.

\subsection{Basis of Multi-Depot Vehicle Routing Problem (MDVRP)}

MDVRP can be considered as an overview of classical VRP. The purpose of this issue is to discover the routes for vehicles to support every one of the clients at a least cost which is as far as number of routes or paths and entire travel distance without disregarding the capacity and travel time restrictions of the vehicles. MDVRP is a NP-hard problem which is more advantageous than VRP which at the same time decides the routes for some vehicles from numerous depots to a set of clients and afterward come back to the same depot. Further, the purpose of MDVRP is to limit the entire delivery distance or time spent in fulfilling the requirements of all consumers. Lower the delivery time, higher the customer loyalty. In spite of the fact that there might 
be several objectives, the intention of MDVRP is to enhance the efficiency of delivery. According to Carlssony et .al (2010), MDVRP is a simplification of the Single-Depot Vehicle Routing Problem (SDVRP) in which vehicle(s) begin from multiple depots and come back to their depots of origin at the end of their appointed tours. The outdated aim in MDVRP is to limit the aggregate of all tour lengths, and prevailing literature handles this issue with an assortment of assumptions and limitations. The MDVRP comprises of structure a set of vehicle routes or paths, so that:

(1) Each and every single route or path starts and finishes at the same depot,

(2) All clients will be visited precisely once by a vehicle,

(3) The absolute demand of every route does not surpass the vehicle capacity,

(4) The entire duration of every route or path (counting travel and service times) does not surpass a preset point of confinement, so that

(5) The entire cost related to routing will be minimized.

\subsection{Exact Methods}

An exact solution technique has being set up by Azi et al.(2010) for the foreseen issue with soft time windows where precise new optimization methodology for the vehicle routing along with scheduling difficulty with soft time windows using new column generation has been suggested . Fundamental level at route with lowest distance issue with the required resource restrictions and penalties for late arrival are replied as a sub issue. This has been used in the Dantzig-Wolfe decomposition method. The author Qureshi (2009) has given a precise methodology for vehicle planning and development issue with soft time windows.

\subsubsection{Set Partitioning (SP) Formulation using algorithms}

(Ballinski \& Quandt ,1964) have suggested a binary variable to signify a feasible route which initially called as SP formulation of CVRP which can be presented as;

Let, ' $\mathrm{R}_{\mathrm{o}}$ '; $\quad$ set of routes

' $\mathrm{r}_{\mathrm{o}}$ '; specific route

$\mathrm{a}_{\text {jro }}=1$ (binary coefficient if and only if vertex $i \in V_{o} \backslash\{0\}$ belongs to path $r_{o}$

$c_{\text {ro }} * ; \quad$ optimum cost of the path ' $\mathrm{r}_{\mathrm{o}}$ '

$y_{k}=1$ (binary variable if and only if route $r_{o}$ is used in optimal solution.

This can be stated as a complete column generation algorithm which has been improved by Agarwal et al.(1989), who answered cases (n) which fallen in the range 15 to 25 . Based on the explaination given by Laporte (2009), it is unrealistic for a direct use of this formulation, due to larger number of possible paths experienced in most non trivial occasions and of the struggle in calculating the $c$ ro $*$ coefficients as it needs answering an exponential number of occasions of NPhard problem.

\subsubsection{Heuristic Approaches}

An effective experiment for route reduction for the foreseen issue has been proposed by Yuichi \& Olli (2009) grounded on discharge pool, strength enclosure along with guided local search techniques. An adaptive parallel plan and the outcomes of broad mathematical experiments introduced by Peng (2011) has demonstrated an efficient and effective route development heuristic. Also heuristics and a scatter search algorithms were suggested by Patricia et at.(2009) which gave the answer for real life heterogeneous vehicle routing issue with time windows along with segmented deliveries.

\subsubsection{Meta Heuristics Approaches}

Parallel calculation methodology is the foundation for most of the fruitful meta-heuristics for all most all VRPTW incidents. VRPTW was applied with a genetic algorithm by Blanton \& Wainwright (1993) where it tested the best ordering frequencies of clients while looking for feasible solution. Apart from Blanton \& Wainwright (1993), hybridization of a genetic algorithm with dissimilar construction heuristics have been represented by Berger (1998), Potvin (1996), Thangiah (1995), Ho (2001) and Berger (2003).

According to Darwin's theory of evolution, developmental algorithms are analysis where the following generation would produce only by the fittest people to populate the following generation. A hybrid ant colony optimization (ACO) was used to tackle VRP in heuristic manner together with algorithm as suggested by Aziz (2010). Geographically distributed clients with known demand were treated with solitary depot using specific set of vehicles in basic VRP. Nodes which are situated close by will likely to have a place with similar branches in spreading tree which is to same as route in VRP and can be recognized as the nature of suggested algorithm. Finally, the answer is to suggest a route in the bunch of customer nodes by utilizing ACO considering the guidelines of the ants. Further, it attempts to enhance the quality of outcomes at the end of each iteration and also to update the related weights of the graph arcs.

A hybridized Ant colony algorithm along with Heuristics for the Time dependent vehicle routing problem with time windows (TDVRPTW) was proposed by Balseiro et. al(2011) which further explained that set of vehicles should carry orders to the clients considering time restrictions where travel time between origin and destination be subject to on the time of leaving.

\section{Conclusion \& Recommendations}

One of the foremost costs that influence the entire cost structure of an organization is the distribution cost. The key activity of properly arranging vehicles and routes can optimize the process of cost reduction. In order to achieve this and deliver goods/ services to customers effectively and efficiently, the supply chain management is an essential component to enhance productivity in organizations. Finding out the latest advancements in Vehicle Routing Problems and its variants are the main objectives of this study. The following areas will precise techniques, heuristics methodologies, meta-heuristics, and hybrid strategies have been focused in the review of literature. The contribution to the field by a number of researchers have also been dealt with at length. It has been found that most of the available literature concentrate on inventory controlling related to inhouse warehousing and mini travel plans and there

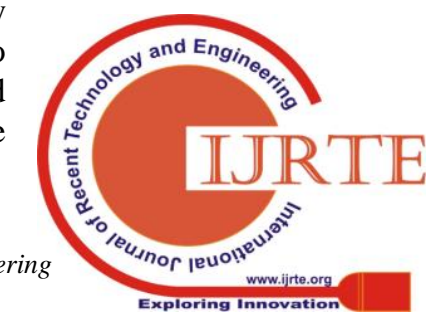


is also necessity for wholistic logistic planning. Most of the researchers have been conducted studies on vehicle routing problems, facility location problems and location routing problems etc. All these issues have been addressed segmentedly like plant to warehouse, warehouse to vendors/retailors, ad then retailors to consumers. Apart from these, it would be much advantageous if future researches to be conducted to cover whole process or to minimize the haulistic process to make the process more efficient. It is recommended for future researchers to address the transportation sector from warehouse to mini hubs and from there to retailers.

\section{REFERENCES}

[1] G. Dantzig and J. Ramser, "The Truck Dispatching Prob- lem," Management Science, Vol. 6, No. 1, 1959, pp. 80-91. doi:10.1287/mnsc.6.1.80

[2] G. Clarke and J. R. Wright, "Scheduling of Vehicle Rout- ing Problem from a Central Depot to a Number of Delivery Points," Operations Research, Vol. 12, No. 4, 1964, pp. 568- 581. doi:10.1287/opre.12.4.568

[3] G. Laporte, "The Vehicle Routing Problem: An Overview of Exact and Approximate Algorithms," European Journal of Operational Research, Vol. 59, No. 3, 1992, pp. 345-358. doi:10.1016/03772217(92)90192-C

[4] M. Gendreau, G. Laporte and J.-Y. Potvin, "Metaheuristics for the VRP," In: P. Toth and D. Vigo, Eds., The Vehicle Routing Problem, SIAM Monographs on Discrete Mathe- matics and Applications, Philadelphia, 2002, pp. 129-154. doi:10.1137/1.9780898718515.ch6

[5] J.-F. Cordeau, M. Gendreau, A. Hertz, G. Laporte and J. S. Sormany, "New Heuristics for the Vehicle Routing Prob- lem," In: A. Langevi and D. Riopel, Eds., Logistics Systems: Design and Optimization, Springer, New York, 2005, pp. 279-297. doi:10.1007/0-387-24977-X_9

[6] M.Goetschalckx,Supply Chain Engineering (Springer Science Business Media LLC,New York, 2011)

[7] Drexl, M. (2012). Rich vehicle routing in theory and practice. Logistics Research, 5(1-2), 47-63.

[8] Toth, P., D. Vigo. (2002). Branch-and-bound algorithms for the capacitated VRP. P. Toth, D. Vigo, eds. The Vehicle Routing Problem.SIAM Monographs on Discrete Mathematics and Applications. Society for Industrial and Applied Mathematics, Philadelphia, 29-51

[9] N. Christofides, S. Eilon , Journal of the Operational Research Society, 1969, Volume 20, Number 3, Page 309

[10] Laporte, G. (2007). What you should know about the vehicle routing problem. Naval Research Logistics, 54 ,

pp. 811-819.

[11] Gendreau, M., Potvin, J. Y. (2010).Handbook of Metaheuristics, Second Edition, Springer, New York.

[12] Laporte, G.,Ropke, S., Vidal, T. (2014). Heuristics for the vehicle routing problem. Vehicle routing:

Problems, Methods and Applications, Second Edition. Philadelphia: SIAM, 18-87

[13] S. Irnich, P. Toth, and D. Vigo. The family of vehicle routing problems. In P. Toth and D. Vigo, editors,
Vehicle routing: Problems, methods, and applications, MOS/SIAM Ser Optim, pages 1-33. 2014. [14]F. Semet, P. Toth, and D. Vigo. Classical exact algorithms for the capacitated vehicle routing problem. In P. Toth and D.

Vigo, editors, Vehicle Routing: Problems, Methods, and Applications, MOS/SIAM Ser Optim, pages 37-58. 2014.

[15] Janacek, J., Janosikova, L., Kohani, M. (2013). Modelovanie a optimalizacia. EDIS vydavatelstvo ZU, in Slovak.

[16] M. Solomon, "Algorithms for the Vehicle Routing and Scheduling Problem with Time Window Constraints,"

Operations Research, Vol. 35, No. 2, 1987, pp. 254265. doi:10.1287/opre.35.2.254

[17] John Carlssony, Dongdong Gez, Arjun Subramaniam, Amy Wu, and Yinyu Ye, " Solving MinMax

Multi-Depot Vehicle Routing Problem" USA publication May 2010

[18] N. Azi, M. Gendreau and J.-Y. Potvin, "An Exact Algo- rithm for a Vehicle Routing Problem with Time Windows and Multiple Use of Vehicles," European Journal of Op- erational Research, Vol. 202, No. 3, 2010, pp. 756-763. doi:10.1016/j.ejor.2009.06.034

[19] A. G. Qureshi, E. Taniguchi and T. Yamada, "An Exact Solution Approach for Vehicle Routing and Scheduling Problems with Soft Time Windows," Transportation Research Part E: Logistics and Transportation Review, Vol. 45, No. 6, 2009, pp. 960977. doi:10.1016/j.tre.2009.04.007

[20] M. L. Ballinski and R. E. Quandt, “On Integer Program for a Delivery Problem," Operations Research, Vol. 12, No. 2, 1964, pp. 300-304. doi:10.1287/opre.12.2.300

[21] Y. Agarwal, K. Mathur and H. M. Salkin, “A SetParti- tioning-Based Algorithm for the Vehicle Routing Problem," Networks, Vol. 19, No. 7, 1989, pp. 731-749. doi:10.1002/net.3230190702

[22] G. Laporte, "Fifty Years of Vehicle Routing," Transportation Science, Vol. 43, No. 4, 2009, pp. 408416.

[23] M. Fisher and R. Jaikumar, "A Generalized Assignment Heuristic for Vehicle Routing," Networks, Vol. 11, No. 2, 1981, pp. 109-124. doi:10.1002/net.3230110205

[24] B. M. Baker and J. Sheasby, "Extensions to the Generalised Assignment Heuristic for Vehicle Routing," European Jour- nal of Operational Research, Vol. 119, No. 1, 1999, pp. 147- 157. doi:10.1016/S03772217(98)00348-8

[25] N. Yuichi and B. Olli, "A Powerful Route Minimization Heuristic for the Vehicle Routing Problem with Time Windows," Operations Research Letters, Vol. 37, No. 5, 2009, pp. 333-338. doi:10.1016/j.orl.2009.04.006

[26] K.-W. Peng, "An Adaptive Parallel Route Construction Heuristic for the Vehicle Routing Problem with Time Windows Constraints," Expert Systems with Applications, Vol. 38, No. 9, 2011, pp. 11939-11946. [27] B. Patricia, T. Hugo and Y. Yoshida, "Scatter 
Search for a Real-Life Heterogeneous Fleet Vehicle Routing Problem with Time Windows and Split Deliveries in Brazil," Euro- pean Journal of Operational Research, Vol. 199, 2009, pp. 750-758. doi:10.1016/j.ejor.2008.08.003

[28] J. L. Blanton and R. L. Wainwright, "Multiple Vehicle Routing with Time and Capacity Constraints Using Ge- netic Algorithms," Proceedings of the 5th International Conference on Genetic Algorithms, San Francisco, 17 July 1993, pp. 452-459.

[29] J. Berger, M. Salois and R. A. Begin, "Hybrid Genetic Algorithm for the Vehicle Routing Problem with Time Windows," Lecture Notes in Artificial Intelligence 1418, Springer, Berlin, 1998, pp. 114-127.

[30] S. R. Thangiah, I. H. Osman and T. Sun, "Hybrid Genetic Algorithms, Simulated Annealing and Tabu Search Meth- ods for Vehicle Routing Problems with Time Windows," Technical Report UKC/OR94/4, Institute of Mathematics and Statistics, University of Kent, Canterbury, 1995.

[31] W.-K. Ho, J. C. Agn and A. Lim, "A Hybrid Search Algo- rithm for the Vehicle Routing Problem with Time Win- dows," International Journal on Artificial Intelligence Tools, Vol. 10, No. 3, 2001, pp. 431-449. doi:10.1142/S021821300100060X

[32] J. Berger, M. Barkaoui and O. A. Bräysy, "RouteDirected Hybrid Genetic Approach for the Vehicle Routing Problem with Time Windows," INFOR, Vol. 41, No. 2, 2003, pp. 179-194.

[33] E. Aziz, "An Algorithm for the Vehicle Problem," Interna- tional Journal of Advanced Robotic Systems, Vol. 7, No. 2, 2010, pp. 125-132.

[34] S. R. Balseiro, I. Loiseau and J. Ramone, "An Ant Colony Algorithm Hybridized with Insertion Heuristics for the Time Dependent Vehicle Routing Problem with Time Windows," Computers \& Operations Research, Vol. 38, 2011, pp. 954-966. doi:10.1016/j.cor.2010.10.011 